\title{
An Improved High-availability Seamless Redundancy (HSR) for dependable Substation Automation System
}

\author{
Huu-Dung Ngo ${ }^{*}$, Hyo-Sik Yang ${ }^{*}$, Dong-Wook Ham ${ }^{*}$, Jongmyung Rhee ${ }^{* *}$, Yongho An ${ }^{\dagger}$, Jeongryul Han ${ }^{\dagger}$, Youjin \\ Lee $^{\dagger}$, Namho Lee ${ }^{\dagger}$ \\ "Department of Computer Engineering, Sejong University, Korea \\ ${ }^{* *}$ Department of Information \& Communication Engineering, Myongii University, Korea \\ ${ }^{\dagger}$ Korea Electric Power Research Institute, Daejun, Korea
}

ngohuudung@hui.edu.vn, hsyang@sejong.edu,donhahm08@gmail.com,jmr77@mju.ac.kr, anyeoh@kepco.co.kr, 06100929@kepco.co.kr, youjinlee@kepco.co.kr, 06101631@kepco.co.kr

\begin{abstract}
High-availability Seamless Redundancy (HSR) is an emerging standard that is suitable for mission-critical communication of IEC 61850 based Substation Automation System in smart grid, where the dependability is very important. This redundancy protocol provides zero recovery time by offering duplicated frames for separate physical paths, but it generates and circulates unnecessary traffic inside the network that will degrade the performance of the network and may cause the congestion, delay, or frame loss. To improve the HSR network performance, the main drawback of generating extra traffic should be considered and eliminated. This paper demonstrates comprehensive performance analysis of IEC 61850 based substation automation networks and applies the quick removing (QR) algorithm to improve the performance of the HSR network to overcome the major drawback. The performance analysis includes the HSR modules, i.e., Doubly Attached Node for HSR (DANH), Redundancy Box (RedBox), and Quadruple Box (QuadBox), and IEC 61850 services, i.e., Generic Object Oriented Substation Events (GOOSE), Sampled Measurement Values (SMV), and report service. Performance analysis was conducted to verify the timing constraints described in IEC 61850 as a reference for designing proper HSR network topology. The numerical results show that the frame loss, restricted in mission-critical network, occurs due to large amount of traffic volume, which is the main drawback of HSR. The QR algorithm improves the traffic performance of HSR by reducing the unnecessary traffic along with the decrease of packet loss significantly.
\end{abstract}

Keywords - Smart grid, IEC 61850, Substation Automation System, High-availability Seamless Redundancy, network performance

\section{INTRODUCTION}

A scalable and pervasive communication infrastructure is essential part for smart grid to enhance efficiency and reliability for a modern electric power grid infrastructure in both construction and operation [1], [2]. The communication network of the Substation Automation System (SAS), defined by IEC 61850 standard, is used to transfer information for monitoring and protecting the primary equipment of inside the substation and its associated feeders [3].

High-availability Seamless Redundancy (HSR), described in IEC 62439-3 clause 5 [4], seamlessly fail-over by applying the principle of frame duplication using ring topology, is an emerging standard that is suitable for mission-critical communication of IEC 61850 based substation automation system in smart grid [5]. The main drawback of HSR is the extra traffic generated due to the redundant frame copies that generated and circulated inside the network that will degrade the network performance and may cause network congestion, delay, or frame drop. Especially with stringent real-time messages such as Generic Object Oriented Substation Events (GOOSE) or Sampled Measurement Values (SMV) which require a large volume of traffic in SAS network, the extra throughput of HSR may lead the network to dropping, restricted in mission-critical network.

Inspired by [6] that the bandwidth of the designed network should meet the requirement and the network traffic should be minimized for efficient use of network bandwidth, and encouraged from a proposed approach at [7] that removes the redundant frame copies to reduce the HSR network traffic, this paper considers the main drawback of generating extra throughput carefully and proposes a concreted solution for improving HSR through a comprehensive performance analysis for IEC 61850 based substation networks. The solution includes abilities to distinguish a frame whether duplicate or not at each HSR node and to remove the unnecessary-duplicated frames. In this paper, performance analysis includes HSR modules such as Doubly Attached Node for HSR (DANH), Redundancy Box (RedBox), Quadruple Box (QuadBox), IEC 61850 services such as GOOSE, SMV, and report service. The numerical results show that the frame loss and the decrease in packet dropping of the proposed solution due to the reducing significantly of unnecessary traffic.

Please direct correspondence to H. S. Yang (hsyang@sejong.edu). 
This paper is structured as follows: The related works are reviewed in Section 2, followed by the HSR implementation in Section 3. Section 4 analysed the simulation results, while Section 5 explained the proposed solution for HSR. Finally, conclusions and future work are given in Section 6.

\section{RELATED WORKS}

A generic modeling technique of intelligent electronic device (IED) and the setup of a research platform using the OPNET Modeler were introduced in [8]. It explained about how to create and setup a platform for the performance study of an IEC 61850-based substation communication system by modeling devices with the specific protocol stack. According to this modeling, Thomas and Ali [9] proposed a network architecture for IEC 61850 substation automation system and demonstrated that it is reliable, fast, and deterministic.

The performance evaluation of the IEC 61850-9-2 process bus for a typical $345 \mathrm{kV} / 230 \mathrm{kV}$ substation by studying the time-critical sampled value messages delay and loss using OPNET simulation tool was presented in [10]. The process bus parameters influenced on the sampled value packet loss and maximum delay. It also proposed the sampled value estimation algorithm for any digital substation relaying and examined it for various power system scenarios with the help of PSCAD/EMTDC and MATLAB simulation tools.

Communication networks and performance analysis were designed and conducted on an IEC 61850 based substation station bus and process bus in [11]. Some network components of IEC 61850 were implemented by adding module for IEC 61850 using NS-2. This module includes the GOOSE, SMV, report, and SNTP time synchronization services, a new trace method to get results and a graphic user interface to make faster and more effective simulation. Three experiments were simulated using this new module on NS-2 including station bus, process bus for transmission line and process bus for distribution line of Poong-Dong digital substation. The performance analysis concluded that the proposed simulation network satisfies the timing requirement of IEC 61850.

A new IEC 61850 simulation platform based on OMMeT++ was also presented at [12]. It does not only allow to conduct a network performance analysis, but also to carry out hardware-in-the-loop simulations before implementing them in to a real device. This platform developed simulation core, which uses two processes working in parallel manner and the implementation of the real IEC 61850 protocol stack.

The generic architecture of IEC 61850 and modelling approach for evaluating high performance and real-time capability of communication technologies in smart grid application are introduced in [13]. The modeling approach was demonstrated and a simulation model was developed along with an analytical approach on the basis of Network Calculus. The results show the applicability of Network Calculus for real-time constrained smart grid communication.

A sequential Monte Carlo method is used to simulate the reliability and probability of failure in [14]. The analytical and simulation solutions show that the redundant ring architecture has increased the reliability compared with the cascaded architecture.

\section{Substation Automation Network}

In this paper, the HSR components such as DANH, RedBox and QuadBox and IEC 61850 services such as GOOSE, SMV, and report service for simulating HSR networks has been implemented using NS-2. A GUI tool based on Java is also implemented to make IEC 61850 based network simulation easier and a result analyser is used to get results efficiently.

\section{A. HSR Components}

HSR uses Doubly Attached Node for HSR (DANH) nodes with two ports, one for clockwise and the other for counterclockwise, to forward frames within HSR network. The Redundancy box (RedBox) is required to attach the HSR network with non-HSR nodes; two Quadboxes are required to connect HSR rings for preventing single point of failure.

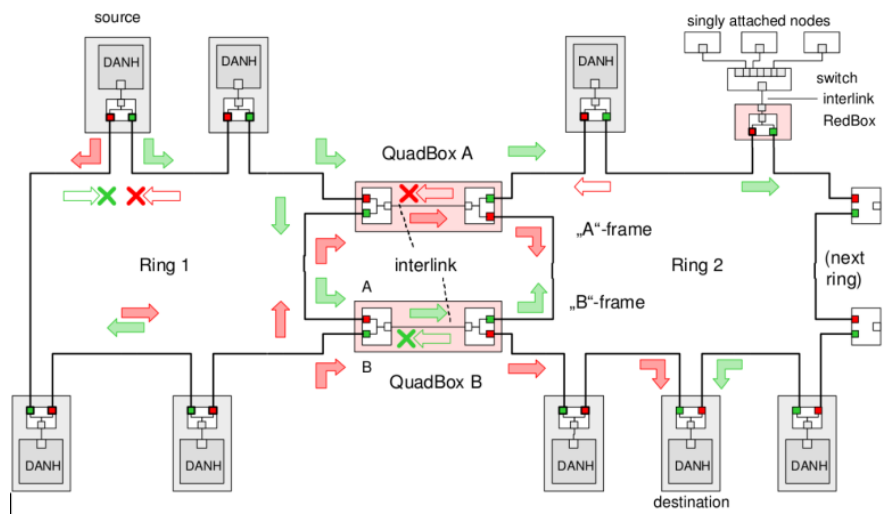

Figure 1. An example of HSR network

Fig. 1 shows an example of a HSR network, consists of DANH, QuadBox, RedBox, and Singly Attached Node (SAN) nodes. Each source DANH inserts a HSR tag to identify frame, duplicates and sends them over its two ports. At destination, DANH receives two identical frames from each port, removes the HSR tag of the first frame, passes it to its upper layer, and forward any duplicate frame to next hop. The SAN nodes cannot be inserted directly into the HSR ring since they have only one port and cannot interpret the HSR tag in the HSR frames. SANs communicate with HSR ring through a RedBox that acts as a proxy for the SANs attached to it. Two HSR rings can be connected using QuadBoxes that forwards frames over each ring as any HSR nodes and pass frames to another ring.

\section{B. IEC 61850 Services and Structure of the Simulator}

GOOSE, SMV, and report services are implemented as applications for generating traffic at HSR components. The GOOSE and SMV services mapped directly to ISO/IEC 88023 Ethernet type with IEEE $802.1 \mathrm{Q}$ priority tagging which reduce the queuing delay generated by passing LLC (Link Layer Control) and ISO transport layer. GOOSE and SMV services are used to deliver time and mission critical messages 
e.g., trip or acquisition messages. These are peer to peer service based multicast services and can be delivered faster than other traffic i.e., MMS (Manufacturing Message Specification) because of priority tagging. MMS using clientserver model, on top of TCP/IP, is used to exchange report, reading and writing variable, and control the device.

The structure of simulator is shown in Fig. 2. There is a GUI based on Java for making simulations easier and generating TCL script automatically. The new module implemented in NS-2 runs the script file from GUI and extracts the simulation result in the new format of trace file. Finally, the trace file can be used to analyse at GUI efficiently.

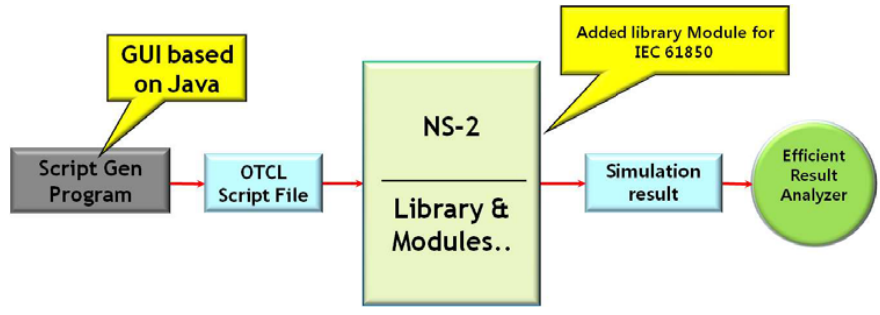

Figure 2. Structure of the simulator

\section{IV.NETWORK SIMULATION}

Various HSR network topologies are considered and the simulation results are analysed in this section. The HSR single ring topology includes Merging Units (MUs) and IED connected into a ring, while the HSR ring of rings topology includes QuadBoxes separating HSR ring into two rings. The worst case of the network, failure of link, is also considered.

\section{A. The HSR Single Ring}

The process bus of the substation is related to gathering measured information, such as voltage, current, and status information from the transformers and transducers connected to the primary power system equipment. Signals from voltage and current transformers are input into a Merging Unit (MU). MUs transmit SMV messages on a multicast mode from one publisher to multiple subscribers. IEDs in same VLAN setup will receive SMV from multiple MUs and automatically align and process the data.

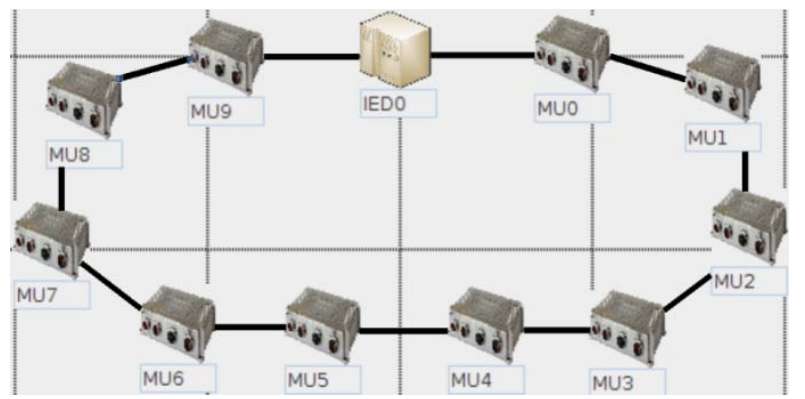

Figure 3. HSR single ring

A HSR ring topology is considered as shown in Figure 3. SMV traffic is generated at a base sample rate of 80 samples per power system cycle for protection and monitoring and at a high rate of 256 samples per power system cycle for high- frequency applications. There are one IED and a number of MUs connected into a HSR ring topology. IED and MUs nodes are applied as DANH nodes in HSR module. The bandwidth of links in Mbps and the number of MUs are varied. SMV message is set to 180 bytes and the interval is varied depending on the rates of sample. The maximum delays in microseconds and the number of frame loss are summarised in Table 1 for both the base and high sample rates.

The maximum delay increases along with the increase of the number of MUs and the decrease of the bandwidth of links. Packet loss occurs under 80 samples/cycle and many loss at 256 samples/cycle. This drop is caused from the main drawback of HSR, extra traffic generated due to the redundant frame copies, making big traffic volume in HSR ring.

TABLE 1. MAXimum Delay AND Number of Drops on HSR Single RING

\begin{tabular}{|c|c|c|c|c|c|c|c|c|c|c|c|}
\hline \multirow{2}{*}{$\begin{array}{l}\text { Samples } \\
\text { /cycle }\end{array}$} & \multirow{2}{*}{$\begin{array}{c}\text { Num. of } \\
\text { MUs }\end{array}$} & \multicolumn{10}{|c|}{ Links (Mbps) } \\
\hline & & 100 & 200 & 300 & 400 & 500 & 600 & 700 & 800 & 900 & 1000 \\
\hline \multicolumn{12}{|c|}{ Maximum delay in microsecond } \\
\hline \multirow{4}{*}{80} & 10 & 73 & 37 & 25 & 19 & 15 & 13 & 11 & 10 & 9 & 8 \\
\hline & 15 & 66132 & 58 & 39 & 30 & 24 & 20 & 17 & 15 & 14 & 12 \\
\hline & 20 & 478098 & 73 & 49 & 37 & 30 & 25 & 22 & 19 & 17 & 15 \\
\hline & 25 & 685964 & 95 & 64 & 48 & 39 & 32 & 28 & 25 & 22 & 20 \\
\hline \multirow{4}{*}{256} & 10 & 806157 & 169482 & 25 & 19 & 15 & 13 & 11 & 10 & 9 & 8 \\
\hline & 15 & 941364 & 643324 & 17445 & 30 & 24 & 20 & 17 & 15 & 14 & 12 \\
\hline & 20 & 977842 & 825761 & 545628 & 177032 & 30 & 25 & 22 & 19 & 17 & 15 \\
\hline & 25 & 987784 & 908740 & 730541 & 481722 & 178641 & 32 & 28 & 25 & 22 & 20 \\
\hline \multicolumn{12}{|c|}{ Number of dropping frames } \\
\hline \multirow{4}{*}{80} & 10 & 0 & 0 & 0 & 0 & 0 & 0 & 0 & 0 & 0 & 0 \\
\hline & 15 & 2187 & 0 & 0 & 0 & 0 & 0 & 0 & 0 & 0 & 0 \\
\hline & 20 & 26164 & 0 & 0 & 0 & 0 & 0 & 0 & 0 & 0 & 0 \\
\hline & 25 & 50098 & 0 & 0 & 0 & 0 & 0 & 0 & 0 & 0 & 0 \\
\hline \multirow{4}{*}{256} & 10 & 84083 & 14540 & 0 & 0 & 0 & 0 & 0 & 0 & 0 & 0 \\
\hline & 15 & 160945 & 91425 & 21893 & 0 & 0 & 0 & 0 & 0 & 0 & 0 \\
\hline & 20 & 237941 & 168431 & 98952 & 29166 & 0 & 0 & 0 & 0 & 0 & 0 \\
\hline & 25 & 314822 & 245390 & 176082 & 106539 & 36469 & 0 & 0 & 0 & 0 & 0 \\
\hline
\end{tabular}

The packet loss is not acceptable in mission critical network and partly caused from the extra traffic of HSR. These simulation results is used for referencing to design HSR ring with suitable number of MUs and the bandwidth of links that satisfying the time constraints. A proposal for HSR, will be introduced in the following subsection, for reducing the traffic, was implemented in the new HSR module and restricted the drop situations significantly.

\section{B. The HSR Ring of Rings}

QuadBox, composed of two Redboxes, is used to connect two HSR rings forming the HSR ring of rings topology. To connect HSR rings redundantly without a single point of failure, two QuadBoxes are installed as shown on Figure 4. Simulations of the HSR ring of rings are the same setup with the HSR single ring simulations but two QuadBoxes are added to separate a HSR single ring into two rings.

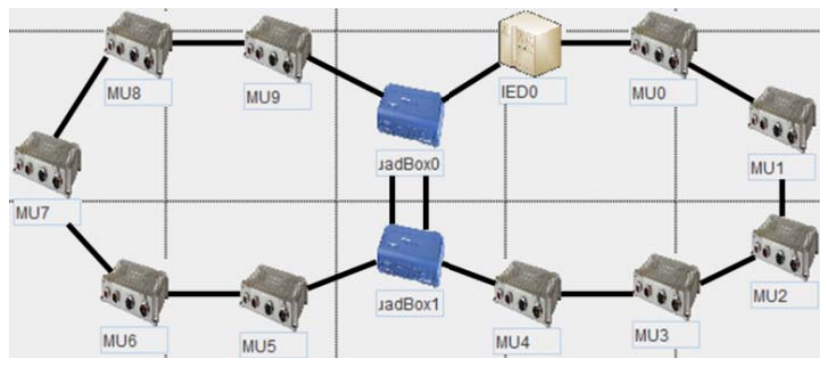

Figure 4. HSR ring of rings 
The maximum delay in micro seconds and the number of dropping frames of HSR ring of rings are shown on Table 2 in two samples rates with the various numbers of MUs and bandwidth of links. The maximum delay at the HSR ring of rings is slightly increased at none frame loss situations comparing to the HSR single ring topology due to the increase of number of nodes and links. Frame loss is the same as HSR single ring topology at some simulations.

TABLE 2. MAXIMUM DELAY AND NUMBER OF DROPS ON HSR RING OF RINGS

\begin{tabular}{|c|c|c|c|c|c|c|c|c|c|c|c|}
\hline \multirow{2}{*}{$\begin{array}{c}\text { Samples } \\
\text { /cycle }\end{array}$} & \multirow{2}{*}{$\begin{array}{l}\text { Num. of } \\
\text { MUs }\end{array}$} & \multicolumn{10}{|c|}{ Links (Mbps) } \\
\hline & & 100 & 200 & 300 & 400 & 500 & 600 & 700 & 800 & 900 & 1000 \\
\hline \multicolumn{12}{|c|}{ Maxim um delay in microsecond } \\
\hline \multirow{4}{*}{80} & 10 & 116 & 58 & 39 & 29 & 24 & 20 & 17 & 15 & 13 & 12 \\
\hline & 15 & 66938 & 87 & 58 & $\frac{29}{44}$ & $\frac{24}{35}$ & 29 & $\frac{11}{25}$ & $\frac{10}{22}$ & $\frac{15}{20}$ & $\frac{12}{18}$ \\
\hline & 20 & 453924 & 116 & 78 & 58 & 47 & 39 & 34 & 30 & 26 & 24 \\
\hline & 25 & 558299 & 167 & 97 & 73 & 58 & 49 & 42 & 37 & 33 & 30 \\
\hline \multirow{4}{*}{256} & 10 & 755187 & 161083 & 44 & 30 & 24 & 20 & 17 & 15 & 13 & 12 \\
\hline & 15 & 910997 & 599994 & 172378 & 51 & 35 & 29 & 25 & 22 & 20 & 18 \\
\hline & 20 & 955687 & 778725 & 481314 & 181772 & 56 & 39 & 34 & 30 & 27 & 24 \\
\hline & 25 & 982425 & 874783 & 647449 & 375066 & 166511 & 58 & 42 & 37 & 33 & 30 \\
\hline \multicolumn{12}{|c|}{ Number of dropping frames } \\
\hline \multirow{4}{*}{80} & 10 & 0 & 0 & 0 & 0 & 0 & 0 & 0 & 0 & 0 & 0 \\
\hline & 15 & 5220 & 0 & 0 & 0 & 0 & 0 & 0 & 0 & 0 & 0 \\
\hline & 20 & 59095 & 0 & 0 & 0 & 0 & 0 & 0 & 0 & 0 & 0 \\
\hline & 25 & 125882 & 0 & 0 & 0 & 0 & 0 & 0 & 0 & 0 & 0 \\
\hline \multirow{4}{*}{256} & 10 & 201344 & 36667 & 0 & 0 & 0 & 0 & 0 & 0 & 0 & 0 \\
\hline & 15 & 336627 & 268849 & 58426 & 0 & 0 & 0 & 0 & 0 & 0 & 0 \\
\hline & 20 & 442310 & 481594 & 421015 & 81231 & 0 & 0 & 0 & 0 & 0 & 0 \\
\hline & 25 & 535746 & 618729 & 702450 & 425233 & 147340 & 0 & 0 & 0 & 0 & 0 \\
\hline
\end{tabular}

\section{The Worst Case}

The same simulations as HSR ring above with various number of MUs and bandwidth of links in two sample rates are conducted but in the worst case that there is a link failure on HSR ring as shown on Fig. 5. This link failure cause the maximum hops between source and destination increase.

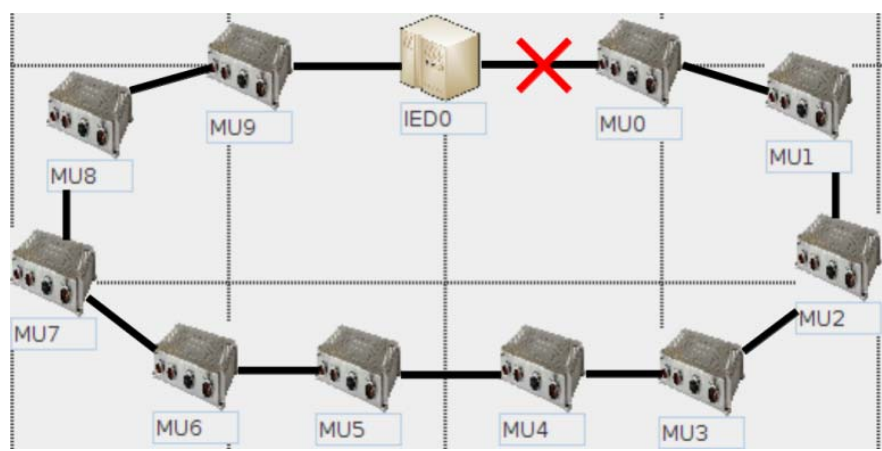

Figure 5. The worst case of HSR single ring

TABLE 3. Simulation Results at the Worst CASE

\begin{tabular}{|c|c|c|c|c|c|c|c|c|c|c|c|}
\hline \multirow{2}{*}{$\begin{array}{l}\text { Samples } \\
\text { /cycle }\end{array}$} & \multirow{2}{*}{$\begin{array}{c}\text { Num. of } \\
\text { MUs }\end{array}$} & \multicolumn{10}{|c|}{ Links (Mbps) } \\
\hline & & 100 & 200 & 300 & 400 & 500 & 600 & 700 & 800 & 900 & 1000 \\
\hline \multicolumn{12}{|c|}{ Maximum delay in microsecond } \\
\hline \multirow{4}{*}{80} & 10 & 145 & 73 & 49 & 37 & 30 & 25 & 21 & 19 & 17 & 15 \\
\hline & 15 & 35698 & 109 & 73 & 55 & 44 & 37 & 32 & 28 & 25 & 23 \\
\hline & 20 & 39686 & 145 & 97 & 73 & 59 & 49 & 42 & 37 & 33 & 30 \\
\hline & 25 & 50715 & 188 & 126 & 92 & 74 & 62 & 53 & 47 & 42 & 38 \\
\hline \multirow{4}{*}{256} & 10 & 667051 & 95842 & 49 & 37 & 30 & 25 & 22 & 19 & 17 & 15 \\
\hline & 15 & 877216 & 464889 & 9778 & 55 & 44 & 37 & 32 & 28 & 25 & 23 \\
\hline & 20 & 953768 & 683320 & 367821 & 98125 & 59 & 49 & 43 & 37 & 33 & 30 \\
\hline & 25 & 980384 & 812581 & 557062 & 310935 & 9872 & 62 & 53 & 47 & 42 & 38 \\
\hline \multicolumn{12}{|c|}{ Number of dropping frames } \\
\hline \multirow{4}{*}{80} & 10 & 0 & 0 & 0 & 0 & 0 & 0 & 0 & 0 & 0 & 0 \\
\hline & 15 & 0 & 0 & 0 & 0 & 0 & 0 & 0 & 0 & 0 & 0 \\
\hline & 20 & 21511 & 0 & 0 & 0 & 0 & 0 & 0 & 0 & 0 & 0 \\
\hline & 25 & 45266 & 0 & 0 & 0 & 0 & 0 & 0 & 0 & 0 & 0 \\
\hline \multirow{4}{*}{256} & 10 & 68547 & 0 & 0 & 0 & 0 & 0 & 0 & 0 & 0 & 0 \\
\hline & 15 & 145097 & 75902 & 6658 & 0 & 0 & 0 & 0 & 0 & 0 & 0 \\
\hline & 20 & 221647 & 152452 & 83208 & 14013 & 0 & 0 & 0 & 0 & 0 & 0 \\
\hline & 25 & 298197 & 229002 & 159758 & 90563 & 21319 & 0 & 0 & 0 & 0 & 0 \\
\hline
\end{tabular}

The maximum delay in micro seconds and the drop problem of HSR single ring simulations at worst case are shown on Table 3. On average, the maximum delay at the worst case is approximately double the maximum delay at normal case on Table 1 since the maximum number of hops between a source and a destination is increased obviously. The frame loss at worst case (Table 3) are sightly reduce comparing with the normal case (Table 1) since the lack of one link cause frames cannot circulate the entire ring.

\section{QUiCK REMOVING AlgorithM}

HSR nodes such as DANH and QuadBox using Quick Removing algorithms are considered to eliminate the wasted traffic in HSR. Simulation analysis for the HSR is also conducted and compared to the standard ones to show the improving of HSR traffic performance.

\section{A. HSR nodes with Quick Removing Algorithm}

Each source HSR node generates two frames of packet, Aframe and B-frame, into two opposite directions of the HSR ring topology. Whenever a destination or an intermediate node has received the first frame from one direction, every node on that direction has received the first frame explicitly. The forwarding of the duplicated frame from opposite direction is not necessary and making wasted traffic.

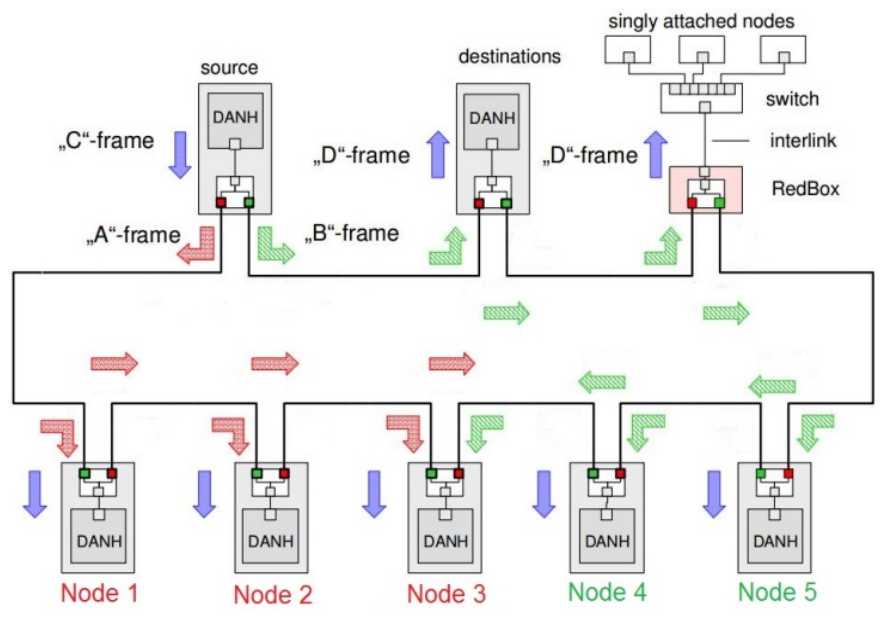

Figure 6. The proposed HSR nodes

As the example on Figure 6, A-frame and B-frame are generated from the source into two directions on the HSR ring. Assuming that A-frame is the first one coming to Node 3 and $\mathrm{B}$-frame is the duplicated one. Whenever Node 3 has received A-frame, Node 1 and Node 2 received A-frame explicitly on that direction, the forwarding of the duplicated B-frame from Node 3 to Node 2 and Node 1 is not necessary.

To eliminate this wasted traffic, the QR HSR nodes adds an ability for stopping to forward the duplicated frame into the direction that has gone through by the first frame. There is a forwarding table at each HSR node that traces packet information for distinguishing whether a coming frame is the first or the duplicate. This table essentially includes the source address and the sequence number of the frame. A frame could be realized as the first or the duplicate by comparing the 
source address and the sequence number information between the frame and the forwarding table.

The forwarding table is updated at the time HSR node receives a frame and its size will be increased along with the increase of the number of received frames. To prevent this increasing, which may lead to a lot of memory and long time of comparing process, the algorithm limit the size of the forwarding table. Each entry of the forwarding table has a tracing time and will be deleted automatically after a certain time-to-live. The number of entries is also limited to a certain value for each source address. Whenever the number of entries of a source address exceed to this value, the oldest one with the smallest sequence number is deleted.

\section{B. Theoretical traffic volume of the HSR network}

The total SMV traffic volume in one second can be expressed as following:

$$
V_{S M V}=F_{S M V} \cdot N_{\text {Frames }} . S_{\text {Frame }}(\mathrm{bps})
$$

Where $F_{S M V}$ is the frequency of SMV traffic, $F_{S M V}=R_{S} \cdot F_{P}$ (Hertz), with $R_{S}$ is the rate of samples and $F_{P}$ is the frequency of power cycle; $N_{\text {Frames }}$ is the number of frames, $N_{\text {Frames }}=2$. Number of $M U s$, since each MU generates two copies of frames; and $S_{\text {Frame }}$ is the size of frame in bit.

On standard HSR ring, two copies of an original frame circulate full ring on two opposite directions as illustrated on Fig. 7. The number of hop that a frame traverse is the double of number of links. As frames generated into two directions, each direction just load a half of SMV traffic volume, then we have the overall throughput at all links on standard HSR ring by the equation (1), where $N_{\text {link }}$ is number of links.

$$
T h r_{-H S R}=2 . N_{\text {link }} . V_{S M V} / 2(\mathrm{bps})
$$

On QR HSR ring, each frame of two copies is transmitted on an opposite direction but not the full ring, since it will be removed any time a frame become duplicated at intermediate nodes. There is one common link that two copies of frame are transmitted as illustrated on Fig. 7. So that, the total number of hops that frames are transmitted is $N_{\text {link }}+1$. Then we have the overall throughput at all link on proposed HSR ring by the equation (2).

$$
T h r_{-Q R} H S R=\left(N_{\text {link }}+1\right) \cdot V_{S M V} / 2(\mathrm{bps})
$$
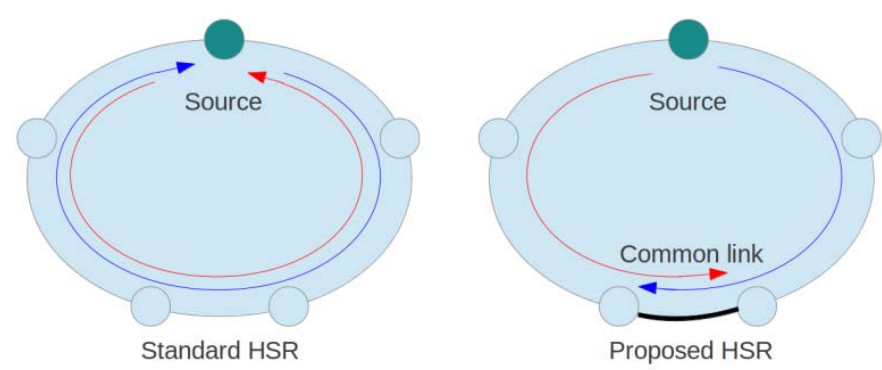

Figure 7. The standard and proposed HSR
From (1) and (2), the overall throughput at the proposed HSR is reduced obviously.

\section{HSR Single Ring using the QR DANH}

Making the same simulations as the HSR single ring at process bus above, but using the DANH with QR algorithm, the maximum delay results in micro second and the number of frame loss are summarized in Table 4. The maximum delay is not different with the HSR single ring that uses standard DANH, but the number of dropping situations is reduced

\begin{tabular}{|c|c|c|c|c|c|c|c|c|c|c|c|}
\hline \multirow{2}{*}{$\begin{array}{c}\text { Samples } \\
\text { Icycle }\end{array}$} & \multirow{2}{*}{$\begin{array}{c}\text { Num. of } \\
\text { MUs }\end{array}$} & \multicolumn{10}{|c|}{ Links (Mbps) } \\
\hline & & 100 & 200 & 300 & 400 & 500 & 600 & 700 & 800 & 900 & 1000 \\
\hline \multicolumn{12}{|c|}{ Maximum delay in microsecond } \\
\hline \multirow{4}{*}{80} & 10 & 73 & 37 & 25 & 19 & 15 & 13 & 11 & 10 & 9 & 8 \\
\hline & 15 & 116 & 58 & 39 & 30 & 24 & 20 & 17 & 15 & 14 & 12 \\
\hline & 20 & 145 & 73 & 49 & 37 & 30 & 25 & 22 & 19 & 17 & 15 \\
\hline & 25 & 188 & 95 & 64 & 48 & 39 & 32 & 28 & 25 & 22 & 20 \\
\hline \multirow{4}{*}{256} & 10 & 805072 & 37 & 25 & 19 & 15 & 13 & 11 & 10 & 9 & 8 \\
\hline & 15 & 940362 & 58 & 39 & 30 & 24 & 20 & 17 & 15 & 14 & 12 \\
\hline & 20 & 979134 & 822963 & 49 & 37 & 30 & 25 & 22 & 19 & 17 & 15 \\
\hline & 25 & 990678 & 906377 & 718988 & 48 & 39 & 32 & 28 & 25 & 22 & 20 \\
\hline \multicolumn{12}{|c|}{ Number of dropping frames } \\
\hline \multirow{4}{*}{80} & 10 & 0 & 0 & 0 & 0 & 0 & 0 & 0 & 0 & 0 & 0 \\
\hline & 15 & 0 & 0 & 0 & 0 & 0 & 0 & 0 & 0 & 0 & 0 \\
\hline & 20 & 0 & 0 & 0 & 0 & 0 & 0 & 0 & 0 & 0 & 0 \\
\hline & 25 & 0 & 0 & 0 & 0 & 0 & 0 & 0 & 0 & 0 & 0 \\
\hline \multirow{4}{*}{256} & 10 & 88383 & 0 & 0 & 0 & 0 & 0 & 0 & 0 & 0 & 0 \\
\hline & 15 & 160854 & 0 & 0 & 0 & 0 & 0 & 0 & 0 & 0 & 0 \\
\hline & 20 & 237895 & 172520 & 0 & 0 & 0 & 0 & 0 & 0 & 0 & 0 \\
\hline & 25 & 314689 & 245273 & 228434 & 0 & 0 & 0 & 0 & 0 & 0 & 0 \\
\hline
\end{tabular}
significantly at both sample rates.

TABLE 4. HSR SINGLE RING USING THE DANH WITH QR ALGORITHM

The average link throughput in Mbps of the HSR ring using the DANH with QR algorithm and the standard DANH is shown on Fig. 8. The average link throughput of the HSR ring using the DANH with QR algorithm accounted for $54.5 \%$ of the ring using standard DANHs at $10 \mathrm{MUs}, 53.1 \%$ at $15 \mathrm{MUs}$ $52.4 \%$ at $20 \mathrm{MUs}$ and $51.9 \%$ at $25 \mathrm{MUs}$ simulations. This is the reason of the decrease on the number of frames loss using the DANH with QR algorithm.

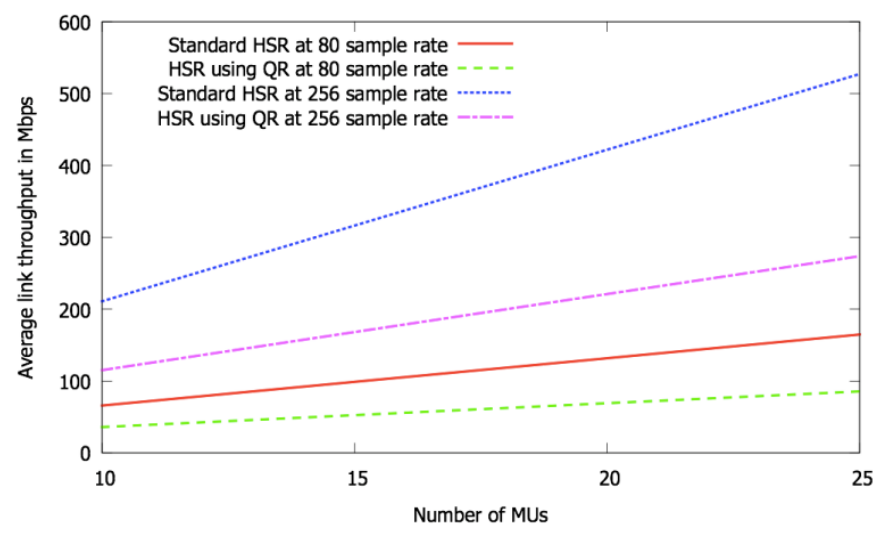

Figure 8. The average link throughput of the HSR single ring using QR algorithm and standard DANH

\section{HSR ring of rings topology}

The same simulation results of HSR ring of rings with QR algorithm are summarized in Table 5 with the maximum delay and the number of frame loss. Maximum delay at none dropping situations is same but the number of drop is reduced 
at Table 5 comparing to Table 2 at the HSR ring of rings topology using the standard HSR nodes.

Fig. 9 shows the average link throughput of the HSR ring of rings topology using HSR with QR algorithm and the standard ones. The average link throughput of the HSR ring of rings topology using the HSR with QR nodes accounted for $57.1 \%$ of the ring using standard ones at $10 \mathrm{MUs}, 55.3 \%$ at $15 \mathrm{MUs}$, $54.2 \%$ at $20 \mathrm{MUs}$, and $53.4 \%$ at $25 \mathrm{MUs}$ simulations.

TABLE 5. HSR RING OF RINGS USING THE PROPOSED HSR NODES
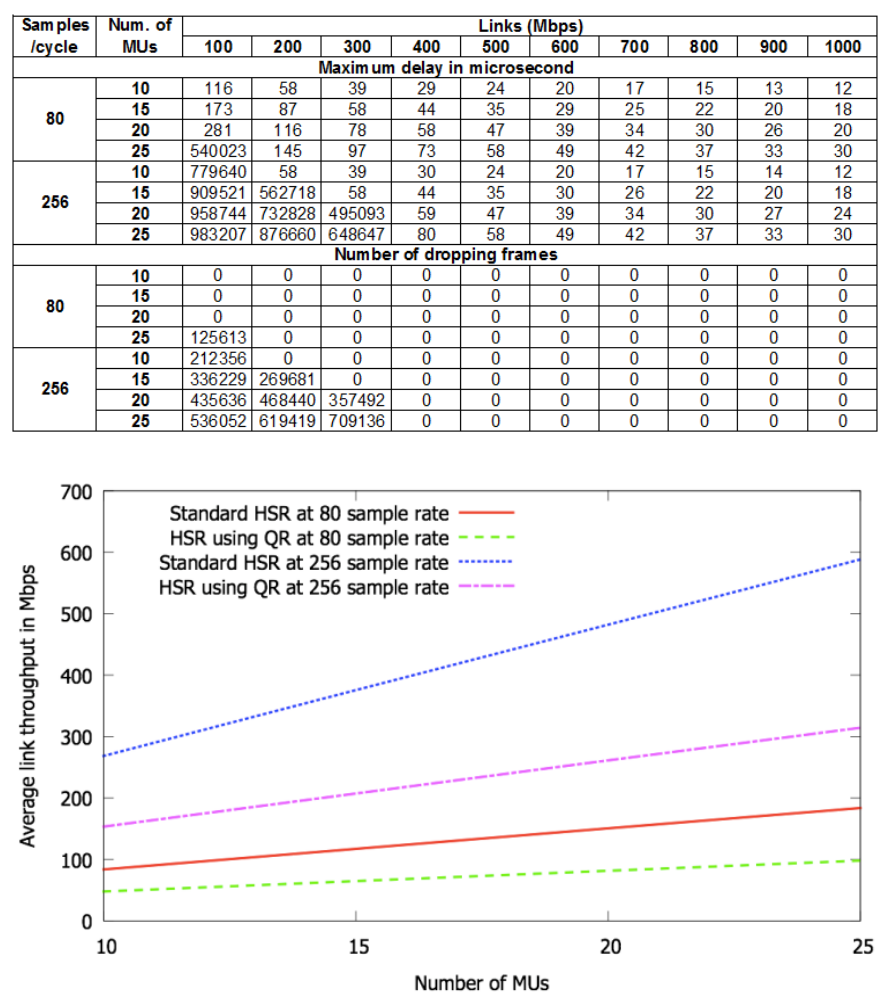

Figure 9. The average link throughput with and without $\mathrm{QR}$ algorithm

\section{VI.CONCLUSION}

HSR is a novel redundancy technology that allows multiple paths from communication source to sink at the same time, enables seamless redundant communication, and prevents looping frames. The main drawback of HSR is, however, the extra traffic since it generates and circulates unnecessary traffic inside the network. This reduces the available usage of bandwidth and influences the HSR network performance.

This paper implemented HSR components such as DANH, QuadBox, RedBox, IEC 61850 services and showed the main drawback of HSR through frame loss. We used Quick Removing algorithm to improve the availability of HSR. We conducted performance analysis to evaluate whether the network topology meets the timing constraints described in the IEC 61850 and provided a reference for designing suitable HSR network topology. The proposed solution improved the traffic performance of HSR, reduced the throughput along with the decrease of packet drop significantly. Based on these initial results, further work includes completed substation automation system performance analysis using HSR and the HSR with QR algorithm in both IEC 61850 based process bus and station bus.

\section{ACKNOWLEDGMENT}

This work was supported by the Human Resources Development program (No. 20134030200310) of the KETEP grant funded by the Korea government Ministry of Trade, Industry and Energy and by Seoul R\&BD Program (SS110012C0214831)

\section{REFERENCES}

[1] C. Gungor, D. Sahin, T. Kocak, S. Ergut, C. Buccella, C. Cecati, and G. P. Hancke, "Smart grid technologies: Communication technologies and standards," IEEE Transactions on Industrial Informatics, vol. 7, no. 4, pp. 529-539, Nov. 2011.

[2] Y. Yan, Y. Qian, H. Sharif, and D. Tipper, "A survey on smart grid communication infrastructures: Motivations, requirements and challenges," IEEE Communications \& Surveys Tutorials, vol. 15, no. 1, pp. 5-20, 2013.

[3] IEC 61850, "Communication networks and system in substation automation," 2002-2005, Available at www.iec.ch.

[4] International Electrotechnical Commission, Geneva "IEC 62439 series: Highly Availability Automation Networks", 2010.

[5] H. Kirrmann, K. Weber, O. Kleineberg and H. Weibel, "Seamless and low-cost redundancy for substation automation systems (High availability Seamless Redundancy, HSR)," IEEE Power and Energy Society General Meeting, pp. 1-7, 2011.

[6] J.C. Tan and W. Luan, "IEC 61850 based substation automation system architecture design," IEEE Power Energy Society General Meeting, pp. 1-6, July 2011.

[7] S.A. Nsaif and J.M. Rhee, "Improvement of high-availability seamless redundancy (HSR) traffic performance for smart grid communications", Journal of Communications and Networks, vol. 14, no. 6, pp. 653-661, Dec. 2012.

[8] T. S. Sidhu and Yujie Yin, "Modeling and simulation for performance evaluation of IEC 61850-based substation communication systems," Power Delivery, IEEE Transactions on, vol. 22, no. 3, pp. 1482-8977, 2007

[9] Mini S. Thomas and Ikbal Ali, "Reliable, fast and deterministic substation network architecture and its performance simulation," Power Delivery, IEEE Transactions on., vol. 25, no. 4, pp. 2364-2370, Oct. 2010.

[10] M.G. Kanabar, T.S. Sidhu, "Performance of IEC 61850-9-2 Process Bus and corrective measure for digital relaying," IEEE Trans. On Power Delivery, vol. 26, no. 2, pp. 725-735, April 2011.

[11] Seung-Ho Yang, Hyo-Sik Yang, Yong-Ho Ahn and Yong-Hak Kim, "Performance Analysis of IEC 61850 based substation," ICACT2012, pp. 854-858, 19-22 Feb. 2012.

[12] Javier Juárez, Carlos R. Morcillo and José A. R. Mondéjar, "Simulation of IEC 61850-based substations under OMNeT++," ICST 2012, pp. 319-326, 19-23 Mar. 2012.

[13] H. Georg, N. Dorsch, M. Putzke, C. Wietfeld, "Performance evaluation of time-critical communication networks for Smart Grids based on IEC 61850," IEEE Conference on Computer Communications Workshops, pp. 43-48, 2013.

[14] M. Mekkanen, R. Virrankoski, M. Elmusrati, E. Antila, "Reliability evaluation and comparison for next-generation substaion function based on IEC 61850 using Monte Carlo simulation," ICCSPA, pp. 1-6, 2013

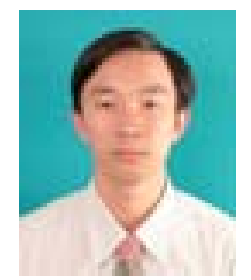

Huu-Dung Ngo received his B. Eng. degree in Electronics and Informatics Engineering, Danang University of Technology, Vietnam in 2005 and M. Eng. degree in Information and Communication Engineering, Sejong University, South Korea in 2007. Currently, he is a Ph.D student in the Department of Computer Engineering at Sejong University, South 
Korea. His research interests are ubiquitous networks and smart grid architecture.

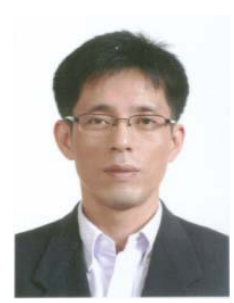

Hyo-Sik Yang (M'98) is an associate professor at the Department of Computer Science and Engineering in Sejong University, Seoul, Korea. He received his B. E. degree in Information and Communication Engineering from Myongji University, Yongin, Korea, in 1998, and his M.S. and Ph.D. in Electrical Engineering from Arizona State University, Tempe, AZ, U.S.A., in 2000 and 2005, respectively. His research interests include wavelength-divisionnetworks, and smart grid. multiplexing all-optical networks, mobile ad-hoc

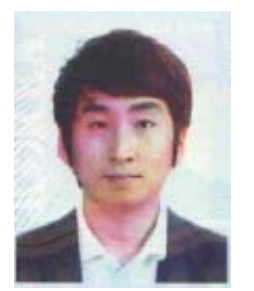

Dong-Wook Ham received B. S. in Department of Computer Science and Engineering from Sejong University, Seoul, Korea in 2013. He is pursuing his M.S. Degree in Sejong University. His research interests include power electronics applications for Substation Automation System complying with IEC 61850, Communication networks and system in substations.

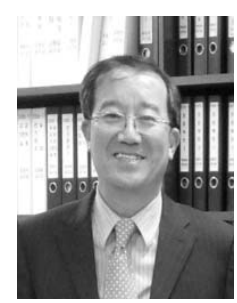

Jong Myung Rhee received his Ph.D. from North Carolina State University, USA, in 1987. After 20 years at the Agency for Defense Development in Korea, where he made noteworthy contributions to C4I and military satellite communications, he joined DACOM and Hanaro Telecom in 1997 and 1999, respectively. At Hanaro Telecom, which was the second largest local carrier in Korea, he served as Chief Technology Officer (CTO), with a senior executive vice-president position. His main duty at Hanaro Telecom was a combination of management and new technology development for high-speed Internet, VoIP, and IPTV. In 2006, he joined Myongji University and is currently a Full Professor in the Information and Communications Engineering Department. His recent research interests are cen- tered on military communications and smart grid, including ad-hoc and fault- tolerant networks. He is a Member of IEEE and IEICE.

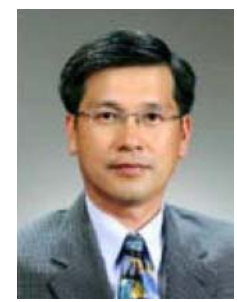

Yong-Ho An received his B.S. degrees in electrical engineering from Inchon University and M.S. in electrical engineering from Chonbuk University in 1984 and 2000, respectively. He has been working as a principal researcher at KEPCO Research Institute since 1995. His research areas include power system protection, IEC 61850 standard substation automation and smart grid.

Jeongryul Han, photograph and biography not available at the time of publication.

Youjin Lee, photograph and biography not available at the time of publication.

Namho Lee, photograph and biography not available at the time of publication. 